\title{
Magnetically Hindered Chain Formation in Transition-Metal Break Junctions
}

\author{
A. Thiess, ${ }^{1, *}$ Y. Mokrousov, ${ }^{1}$ S. Heinze, ${ }^{2}$ and S. Blügel ${ }^{1}$ \\ ${ }^{1}$ Institut für Festkörperforschung and Institute for Advanced Simulation, Forschungszentrum Jülich, D-52425 Jülich, Germany \\ ${ }^{2}$ Institut für Theoretische Physik und Astrophysik, Christian-Albrechts-Universität zu Kiel, D-24098 Kiel, Germany
}

(Received 30 June 2009; published 16 November 2009)

Based on first-principles calculations, we demonstrate that magnetism impedes the formation of long chains in break junctions. We find a distinct softening of the binding energy of atomic chains due to the creation of magnetic moments that crucially reduces the probability of successful chain formation. Thereby, we are able to explain the long standing puzzle why most of the transition metals do not assemble as long chains in break junctions and thus provide indirect evidence that in general suspended atomic chains in transition-metal break junctions are magnetic.

PACS numbers: 75.75.+a, 73.63.Rt

One-dimensional systems, realized experimentally as suspended monatomic chains in break junctions (BJs), have altered our conceptional view on atomic scale junctions. For example, because of their enhanced tendency to magnetism they bear high potential in the field of spintronics by combining the possibility to probe, control [1,2], and switch the magnetic state by spin-polarized electrical currents. Recent first-principles calculations support these expectations and report on sizable magnetic moments [37] and giant magnetocrystalline anisotropy energies [4,5,7] in suspended and free-standing transition-metal (TM) monowires (MWs). While the formation of long atomic chains of selected TMs and conductance quantization have been experimentally demonstrated [8-10], any conclusive evidence of magnetism in chains is still missing.

This lack of evidence seems even more surprising as measurements on chains deposited on surfaces show univocal signatures of local magnetic moments [11,12]. Measuring the magnetoresistance in BJs would serve as a proof for magnetism in atomic-sized contacts. In materials, where long MWs can be successfully suspended as reported for Ir and Pt $[9,13,14]$, the leads are nonmagnetic, which prevents the pinning tip-magnetization and thus the analysis via magnetoresistivity measurements. On the other hand, so far it has not been shown that breaking contacts of magnetic $3 d$-TMs results in one-dimensional structures beyond point contacts.

An alternative approach to prove that monatomic chains are magnetic is the search for half-integer conductance originating from $100 \%$ spin-polarized conductance channels. Although Rodrigues et al. [15] reported on such a half-integer conductance for $\mathrm{Co}, \mathrm{Ni}$ and $\mathrm{Pt} \mathrm{BJ}$ s, it was shown both experimentally [16] and theoretically [17] that not only magnetic ordering but, e.g., also the adsorption of $\mathrm{H}_{2}$ can lead to similar values of conductance. In other words, the presence of half-integer conductance is not a unique attribute of full spin-polarization and thus cannot serve as a proof of it.

Because of these uncertainties, in this Letter we take a new path to address the emergence of magnetism in BJs.
We base our study on the most fundamental and easiest accessible experimental quantity: the probability for successful chain formation of a given material itself. The trend arising from numerous $\mathrm{BJ}$ experiments is that monatomic chain formation is most probable for late $5 d \mathrm{TMs}$ as well as $\mathrm{Ag}$ and $\mathrm{Au}[10,13,14,18]$. To analyze the role of magnetism for the chain formation in BJs of $3 d, 4 d$, and $5 d$ TMs we apply a recently developed material-specific theoretical model [19] for the formation of long monatomic chains which operates in terms of parameters extracted from $a b$ initio calculations. By explicitly including and excluding magnetic exchange interactions, we prove that magnetism significantly suppresses the chain formation due to a substantial reduction of the chain hardness expressed in terms of the maximally sustainable break force. Comparing our results to experimental findings, we are able to provide an indirect evidence that chains in BJs are indeed magnetic.

We will briefly recall the model which allows us to investigate the formation probability of suspended monatomic chains under tension in BJs [19] before applying it to both magnetic and nonmagnetic chains. In this model, chain formation succeeds if the criteria for stability and producibility are met. The criterion for stability addresses the rupture of the chain via breaking of the bond between two neighboring chain atoms. The criterion for producibility is concerned with the chain elongation that is composed of two processes: At first one atom has to be extracted out of the lead into the chain. This transfer leads to a reduction of the coordination of this particular atom and consequently additional external energy is required, which we account for by the difference $\Delta E_{\text {Lead }}=E_{\mathrm{MW}}-$ $E_{\text {Lead }}$ of the energy of cohesion for an atom in the lead, $E_{\text {Lead }}$, and in the chain, $E_{\mathrm{MW}}$, both at equilibrium distance. This energy can be stored mechanically and is released in the second process of relaxing all chain's bonds to a smaller interatomic distance after an additional atom has joined the chain. These competing contributions to the total energy of the system determine whether it is energetically favorable to grow the chain by one atom or not. In order to 
apply both criteria only two quantities have to be known: the binding energy $\mathcal{E}(d)$ of a MW atom as a function of interatomic distance $d$ and the cohesive energy difference $\Delta E_{\text {Lead }}$. We will show that both parameters depend on the formation of magnetic moments.

In order to determine these parameters we carried out spin-polarized and non-spin-polarized $a b$ initio calculations in the generalized gradient approximation [20] to the density functional theory for selected $3 d, 4 d$, and $5 d$ TMs, employing the full-potential linearized augmented plane-wave method for one-dimensional (1D) systems [21], as implemented in the FLEUR code [22]. We used the bulk version of this code to calculate the cohesion energy [23]. Here we considered in all cases the true magnetic $(3 d)$ or nonmagnetic $(4 d, 5 d)$ bulk ground state as the reference configuration. For calculations of the bare MWs in all cases we considered the non- (NM), ferro(FM) and antiferromagnetic (AFM) order and included basis functions with plane waves up to $k_{\max }=4.4$ a.u. $^{-1}$ and used $64 k$ points in one-half of the 1D Brillouin zone. We calculated all $3 d$ MWs in the scalar-relativistic approach, while spin-orbit coupling was added for all $4 d$ 's and $5 d$ 's.

The calculated spin moments $M(d)$ and the binding energy $\mathcal{E}(d)$ are shown in Fig. 1 for Fe and W MWs as examples. Both chains exhibit sizable magnetic moments and the binding energies for the nonmagnetic (NM) and magnetic (M) case differ substantially. This is true, in particular, for large distances, where the energy difference $\mathcal{E}_{\mathrm{sp}}(d)=\mathcal{E}_{\mathrm{NM}}(d)-\mathcal{E}_{\mathrm{M}}(d)$ approaches the spinpolarization energy $\mathcal{E}_{\text {sp }}(\infty)$ of an isolated atom. A closer look reveals, that this overall tendency to magnetism in $1 \mathrm{D}$

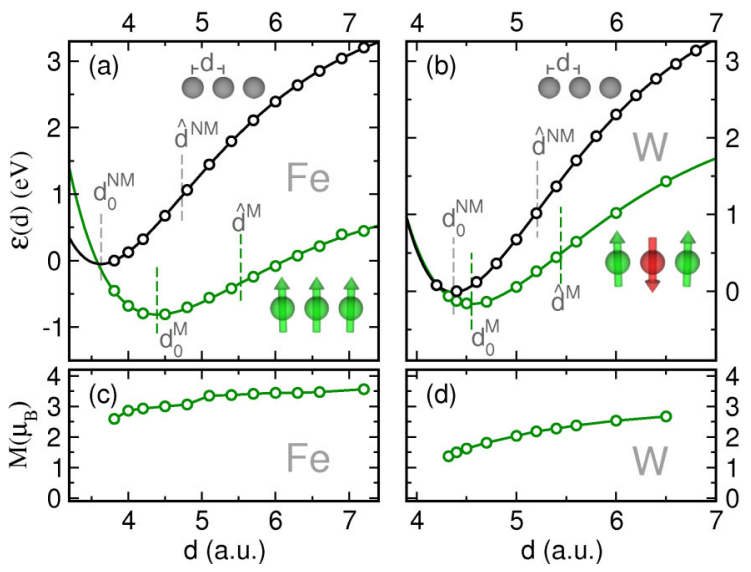

FIG. 1 (color online). Calculated MW energy $\mathcal{E}(d)$ (circles) as a function of interatomic distance $d$ (throughout the Letter given in a.u. $=0.0529 \mathrm{~nm}$ ) for both nonmagnetic (NM) (black) and magnetic (M) state (green or gray) are shown for (a) $\mathrm{Fe}$ and (b) W. The Morse fit (lines) provides a universal fit to these points well below the required accuracy of about $100 \mathrm{meV}$ for chemical bonding and can be characterized by the equilibrium interatomic distance $d_{0}$ and the inflection point $\hat{d}$. $\mathcal{E}(d)$ and the magnetic moments $M$ in (c) and (d) correspond to the FM and AFM ground state for $\mathrm{Fe}$ and $\mathrm{W}$, respectively. chains results in finite magnetic moments even in $5 d$-TMs already at the NM equilibrium distance $d_{0}^{\mathrm{NM}}$ or upon small stretching leading to a magnetic expansion of, e.g., $d_{0}^{\mathrm{M}}-$ $d_{0}^{\mathrm{NM}}=0.2$ a.u. for $\mathrm{W}$ [Fig. 1(a)] and being more pronounced, e.g., for Fe [Fig. 1(b)], in agreement with Refs. [5,25]. Overall, the binding potential energies of $M$ and NM chains differ not only by spin-polarization energy and a constant shift in $d_{0}$, but also their slopes are crucially different [Figs. 1(a) and 1(b)].

In order to analyze the binding energy quantitatively, we fit a Morse potential

$$
\mathcal{E}(d)=\mathcal{E}(\infty)\left(1-e^{-\gamma\left(d-d_{0}\right)}\right)^{2}
$$

to the discrete set of calculated energy points [Figs. 1(a) and 1(b)] for the different magnetic states. Besides the equilibrium distance $d_{0}$, the Morse potential can be characterized by the following two physically transparent parameters: the inflection point $\hat{d}=d_{0}+\ln 2 / \gamma$ and the break force $F_{0}=\gamma \mathcal{E}(\infty) / 2$, which is the maximal slope $F(\hat{d})$ of the potential. Together with $\Delta E_{\text {Lead }}$ we evaluate for a given close-packed surface [Fig. 2(a)], these four quantities constitute a minimal basis for a realistic description of the chain formation process, providing us with an accurate and continuous representation of the binding energy curve.

As we see from Fig. 2, magnetism has a very different impact on these four quantities: The cohesion energy difference $\Delta E_{\text {Lead }}$ [Fig. 2(a)] of $4 d$ and $5 d$ TMs remains almost unchanged for magnetic and nonmagnetic chains, as these elements are nonmagnetic at surfaces and develop only small magnetic moments at $d_{0}$. For $3 d$ TMs the situation is different: in the wire the magnetic moments at $d_{0}$ are already close to the saturated values and larger than for atoms at surfaces, which leads to a sizable gain in spin-polarization energy and thereby to a considerable reduction of $\Delta E_{\text {Lead }}$ [26]. The position of $\hat{d}$ with respect to $d_{0}$ [Fig. 2(b)], a quantity crucial for the stability of the wires, reveals only minor changes of less than $5 \%$ between calculations with and without spin polarization. The crucial impact of magnetism is the softening of the binding energy curve, which leads to a significant reduction of the break force $F_{0}$ for all considered $3 d, 4 d$ (not shown) and $5 d$ TMs [Fig. 2(c)]. For example, the break forces with and without spin-polarization for W differ by a factor of 2 . Both at the beginning and the end of the $5 d$ series, this ratio reduces according to smaller maximal magnetic moments and is equal to one for the nonmagnetic noble metal Au. The break force $F_{0}$ not only represents the maximal force applicable to a chain, but also serves as a measure for the maximal amount of mechanical energy which can be gained upon relaxation of its bonds. In any case, high values of $F_{0}$ favor successful chain creation in BJ experiments. Thus, as the appearance of magnetism crucially reduces $F_{0}$, we can conclude that the formation of local magnetic moments suppresses chain formation. 

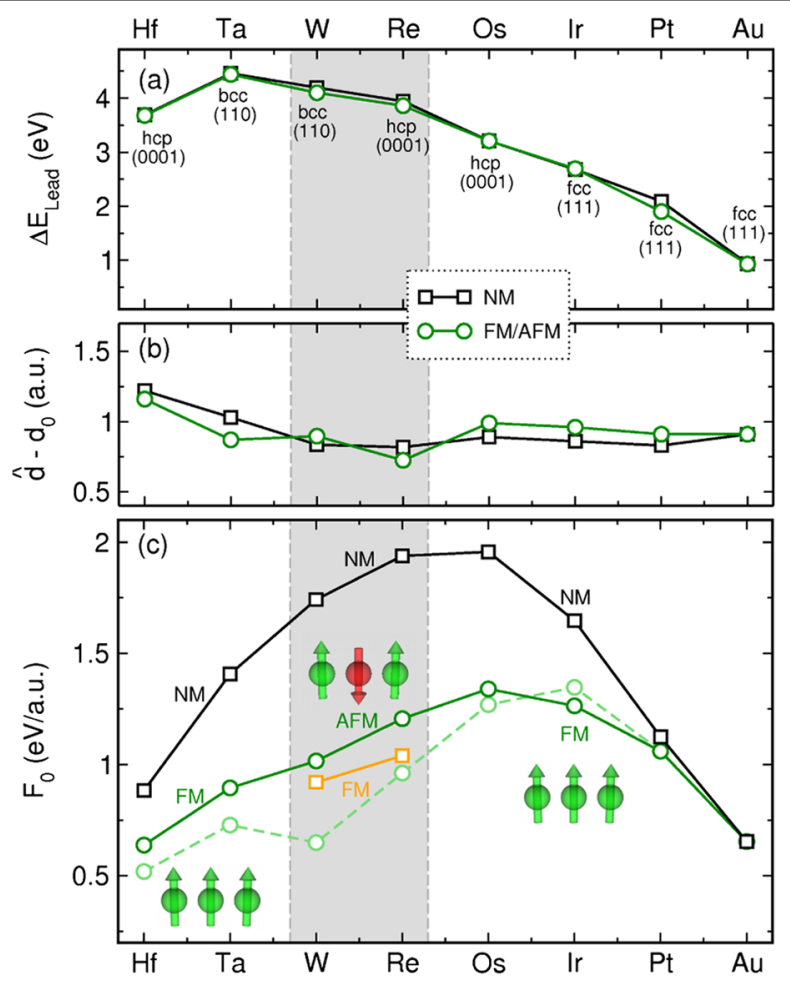

FIG. 2 (color online). (a) Cohesion energy difference $\Delta E_{\text {Lead }}$, (b) difference $\hat{d}-d_{0}=\ln 2 / \gamma$ and (c) break force $F_{0}$ for nonmagnetic (marked as NM, black squares) and magnetic (marked as FM or AFM, green circles) $5 d$-TM chains. Broken line in (c) stands for the break force $F_{0}^{\mathrm{M}}$ calculated according to Eq. (2). As indicated by the gray shaded area, W and Re MWs reveal an AFM ground state at all interatomic distances, while the rest of the chains are FM. With orange squares in (c) the break force for the FM W and Re MWs is shown for comparison.

In order to capture the origin of the magnetically induced reduction of $F_{0}$, we relate the magnetic quantities to the nonmagnetic ones. In the first approximation, the influence of the magnetization on the binding energy potential $\mathcal{E}_{\text {sp }}(d)=\mathcal{E}_{\mathrm{NM}}(d)-\mathcal{E}_{\mathrm{M}}(d)$ can be attributed to the Hund-type intra-atomic exchange interaction $\frac{1}{2} I M^{2}(d)$ between mostly $d$ electrons but also $s$ electrons on the atomic sites and the interatomic Heisenberg $J(d) \vec{M}_{i}(d) \vec{M}_{i+1}(d)$ exchange contributions between the atomic spins. The binding energy, $\mathcal{E}_{\mathrm{M}}(\infty)$, prefactor in Eq. (1), then reads $\mathcal{E}_{\mathrm{M}}(\infty)=\mathcal{E}_{\mathrm{NM}}(\infty)-\mathcal{E}_{\mathrm{sp}}(\infty)+\mathcal{E}_{\mathrm{sp}}\left(d_{0}\right)$, where $\mathcal{E}_{\mathrm{sp}}(\infty)=$ $\frac{1}{2} I M^{2}(\infty)$ is purely given by intra-atomic exchange of a free atom and $\mathcal{E}_{\mathrm{sp}}\left(d_{0}\right)=\mathcal{E}_{\mathrm{NM}}\left(d_{0}^{\mathrm{NM}}\right)-\mathcal{E}_{\mathrm{M}}\left(d_{0}^{\mathrm{M}}\right)$ is the energy difference of the NM and M states at the corresponding equilibrium distance. Taking as an example $5 d$ TMs we can safely assume even for $\mathrm{W}$, exhibiting the largest equilibrium magnetic moment of $M\left(d_{0}^{\mathrm{M}}\right) \approx 1 \mu_{B}$ through the series, that the impact of magnetism on the equilibrium properties of the chains is small and set for simplicity $d_{0}^{\mathrm{M}}=$ $d_{0}^{\mathrm{NM}}$ and $\mathcal{E}_{\text {sp }}\left(d_{0}\right)=0$. As is apparent from Fig. 2(b), it is also reasonable to assume that $\gamma_{\mathrm{M}}=\gamma_{\mathrm{NM}}$, in which case the magnetic break force simplifies to

$$
F_{0}^{\mathrm{M}}=\frac{\gamma_{\mathrm{M}}}{2} \mathcal{E}_{\mathrm{M}}(\infty)=F_{0}^{\mathrm{NM}}-\frac{\gamma_{\mathrm{NM}}}{2} I_{d} M^{2}(\infty)
$$

For $5 d$ TM chains we estimated $F_{0}^{\mathrm{M}}$ according to Eq. (2) using ab initio values of the nonmagnetic break force $F_{0}^{\mathrm{NM}}$, magnetic moments at $d=6.5$ a.u. and the atomic exchange integrals between $d$ electrons, $I_{d}$, from Ref. [27], and plotted it in comparison to the magnetic break force
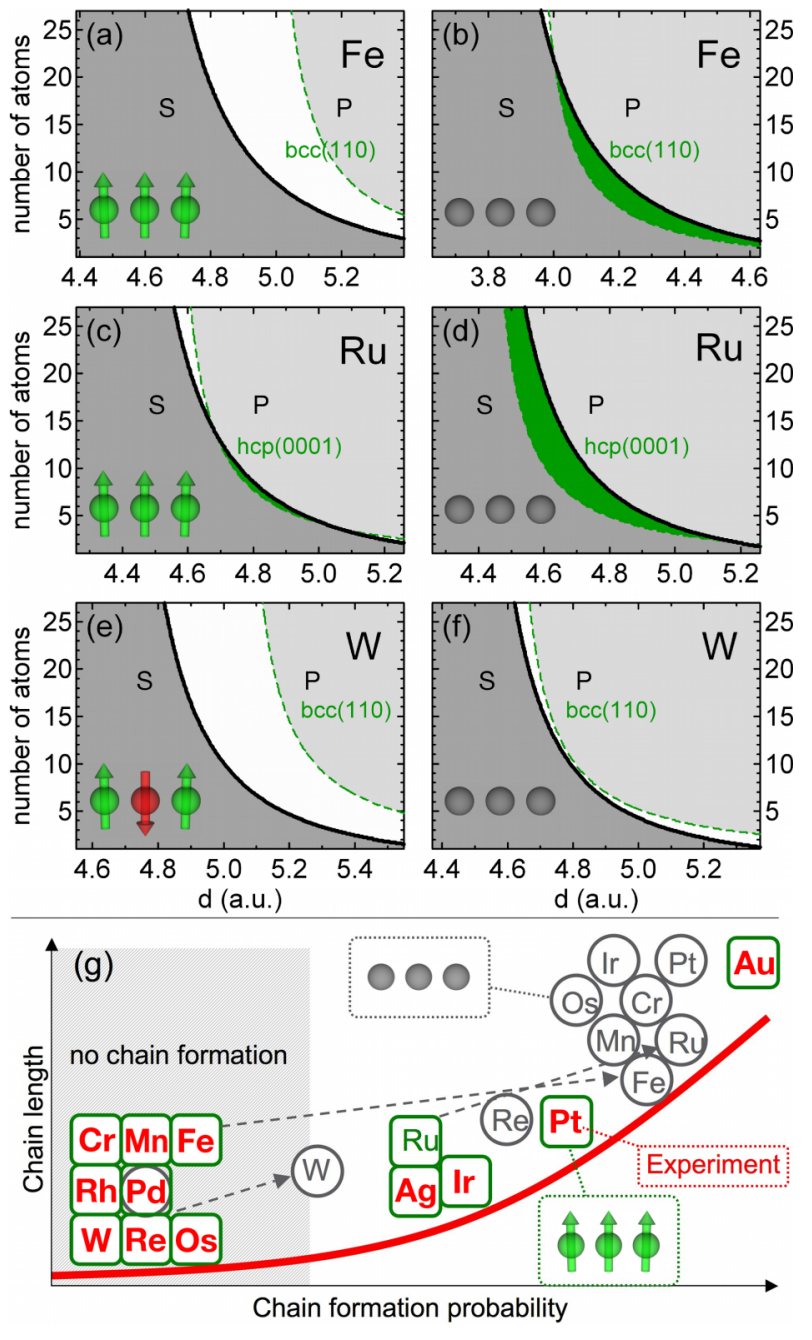

FIG. 3 (color online). Phase diagrams for Fe, Ru, and W-BJs with (a), (c), (e) and without (b), (d), (f) spin polarization of the chain atoms. Plots indicate regions of stability ( $S$, gray), producibility $(P$, light gray), separated by a white region or overlapping in the $S P$ region (in green or dark gray). The starting point along the $x$ axis is $d_{0}$ and the assumed surface orientations of the leads are indicated. The input parameters for $\mathrm{W}$ are given in Figs. 2(a)-2(c) and for $\mathrm{Fe}$ and $\mathrm{Ru}$ in [26]. (g) provides a schematic summary of our results. Shown is the chain formation probability, proportional to the size of $S P$ regions, versus chain length, proportional to the highest $N$ for which an $S P$ region exists, with (green boxes) and without spin polarization (gray circles) in relation to the experimental findings (bold red) $[10,13,14,16,18,29]$ in all cases in arbitrary units relative to $\mathrm{Au}$. Arrows indicate (exemplified for $\mathrm{Fe}, \mathrm{Ru}$, and $\mathrm{W}$ ) the consequence on the chain formation caused by switching off the finite magnetization in the chain. 
determined from $a b$ initio in Fig. 2(c). Good qualitative agreement between the two break force curves underlines that the intra-atomic exchange $I$ is the major origin of the magnetically driven reduction of $F_{0}$, while the interatomic exchange plays only a minor role. This conclusion is further verified by the observation that changing the magnetic order from AFM to FM in W and Re MWs results only in a small change of $F_{0}$ [see Fig. 2(c)].

We now turn back to the analysis of our $a b$ initio results. With the knowledge of all key quantities entering the criteria for stability and producibility we can further analyze both criteria in the phase space of the number of atoms $N$ and interatomic distance $d$ presented in Fig. 3. Each of the criteria leads to a distinct region where it is fulfilled and accordingly the chain is stable $(S)$ or producible $(P)$. Ideally for a successful chain elongation event to happen, both regions $(S)$ and $(P)$ have to overlap $(S P)$.

Comparison of the phase diagrams for different TMs, shown for $\mathrm{Fe}, \mathrm{Ru}$, and $\mathrm{W}$ in Fig. 3, underlines, that the formation of local magnetic moments strongly suppresses the probability of chain formation for $3 d, 4 d$, and $5 d$ elements. If we ignore the formation of magnetism among the $3 d-, 4 d$-, and $5 d$-TM series $\mathrm{Cr}, \mathrm{Mn}, \mathrm{Fe}, \mathrm{Ru}, \mathrm{Rh}, \mathrm{Ag}, \mathrm{Re}$, $\mathrm{Os}$, Ir, Pt, and Au exhibit extensive $S P$ regions [Figs. 3(b) and 3(d)] indicating successful chain formation for these elements. Even for W [Fig. 3(f)] with bcc(110) electrodes neglecting magnetism results in touching $S$ and $P$ regions, indicating chain formation for more open lead structures. Allowing for the formation of local spin moments the picture changes completely: $S P$ regions emerge exclusively for Ru [Fig. 3(c)], Ag, Ir, Pt, and Au while for all other elements the $S$ and $P$ regions are clearly separated and no chain formation occurs [Figs. 3(a) and 3(e) and in [19] ]. While for Pd and Pt chains the influence of magnetism is small due to relatively small moments entering Eq. (2), the $S P$ regions of Ru [Fig. 3(c)] and Ir are considerably less extended for magnetic chains than for nonmagnetic ones, underlining the suppression of chain formation by magnetism.

While our predictions based on the assumption that chains in BJs are magnetic match and explain the experimental findings for successful $\mathrm{Ag}$, Ir, Pt, and $\mathrm{Au}$ chain formation, the results of the model for nonmagnetic suspended chains contradict the experimental observations at several crucial points [Fig. 3(g)]. First, nanocontacts of $3 d$-TMs such as Fe are reported to form only point contacts with no tendency to form longer chains [16]. Second, also BJ experiments using $\mathrm{W}$ as tip material result only in point contacts [28], moreover, W tips are widely used in STM and AFM experiments due to their structural rigidity preventing substrate-induced reformations [29]. Third, nonmagnetic Ir chains would become as long as those of Pt, and almost as long as those of $\mathrm{Au}$, in direct disagreement with experiments which report significant decrease of chain formation probability and length when going from $\mathrm{Au}$ to $\operatorname{Ir}[10,13,14,18]$. These clear contradictions to exist- ing experimental evidence lead us to the conclusion that only when chains in BJs are magnetic, the experimentally observed trends can be reproduced and explained throughout the $3 d, 4 d$, and $5 d$ transition- and noble-metal series. Therefore, by reductio ad contradictum, comparing theoretical predictions with experimental findings, we provide a convincing evidence that TM chains in BJs are magnetic.

We thank J. M. van Ruitenbeek for fruitful discussions. S.H. thanks the Stifterverband für die Deutsche Wissenschaft for financial support.

*a.thiess@fz-juelich.de

[1] P. Lucignano et al., Nature Mater. 8, 563 (2009).

[2] M. R. Calvo et al., Nature (London) 458, 1150 (2009).

[3] A. Delin and E. Tosatti, Phys. Rev. B 68, 144434 (2003).

[4] Y. Mokrousov et al., Phys. Rev. Lett. 96, 147201 (2006).

[5] J. C. Tung and G. Y. Guo, Phys. Rev. B 76, 094413 (2007).

[6] J. Fernández-Rossier et al., Phys. Rev. B 72, 224418 (2005).

[7] A. Smogunov, A. Dal Corso, and E. Tosatti, Phys. Rev. B 78, 014423 (2008).

[8] A. I. Yanson et al., Nature (London) 395, 783 (1998).

[9] R. H. M. Smit et al., Phys. Rev. Lett. 87, 266102 (2001).

[10] T. Kizuka, Phys. Rev. B 77, 155401 (2008).

[11] P. Gambardella et al., Nature (London) 416, 301 (2002).

[12] C. F. Hirjibehedin, C. P. Lutz, and A. J. Heinrich, Science 312, 1021 (2006).

[13] M. Ryu and T. Kizuka, Jpn. J. Appl. Phys. 45, 8952 (2006).

[14] T. Shiota et al., Phys. Rev. B 77, 125411 (2008).

[15] V. Rodrigues et al., Phys. Rev. Lett. 91, 096801 (2003).

[16] C. Untiedt et al., Phys. Rev. B 69, 081401(R) (2004).

[17] V. M. García-Suárez et al., Phys. Rev. B 72, 045437 (2005).

[18] W.H. A. Thijssen et al., Phys. Rev. Lett. 96, 026806 (2006).

[19] A. Thiess et al., Nano Lett. 8, 2144 (2008).

[20] J. P. Perdew, K. Burke, and M. Ernzerhof, Phys. Rev. Lett. 77, 3865 (1996).

[21] Y. Mokrousov, G. Bihlmayer, and S. Blügel, Phys. Rev. B 72, 045402 (2005).

[22] http://www.flapw.de.

[23] We calculate $\Delta E_{\text {surf }}$ by substracting the surface energy of the corresponding element from Ref. [24] from the calculated energy difference $\Delta E_{\text {bulk }}$ between a bulk atom and a MW atom at $d_{0}$.

[24] H. L. Skriver and N. M. Rosengaard, Phys. Rev. B 46, 7157 (1992).

[25] D. Spišák and J. Hafner, Phys. Rev. B 67, 214416 (2003).

[26] See EPAPS Document No. E-PRLTAO-103-034947 for the set of parameters obtained for $\mathrm{Fe}$ and $\mathrm{Ru}$ chains. For more information on EPAPS, see http://www.aip.org/ pubservs/epaps.html.

[27] M. S. S. Brooks and B. Johansson, J. Phys. F 13, L197 (1983).

[28] A. Halbritter et al., Phys. Rev. B 68, 035417 (2003).

[29] U. Landman et al., Science 248, 454 (1990). 